\title{
Qualified Breakdown of Wi-Fi with and without standard in Wireless Networks - Onus on Throughput
}

\author{
Bhat Geetalaxmi Jairam ${ }^{1}$, D. V. Ashoka ${ }^{2}$ \\ Department of Information Science and Engineering, National Institute of Engineering, Mysore, Karnataka, India ${ }^{1}$ \\ Department of Computer/ Information Science and Engineering, JSS Academy of Technical Education, Banglore,
}

$$
\text { Karnataka, India }{ }^{2}
$$

\begin{abstract}
Wireless networking equipments available supports varying levels of industry communication standards. At present the IEEE $802.11 \mathrm{~b} / \mathrm{g}$ standards are widely accepted throughout the industry and provide the necessary balance of range, network throughput and support for device mobility to effectively serve most needs of university community. The authors have concentrated on through put of different networks consisting of 4,5,6 and 7 nodes. Authors are trying to justify that applying standards to wireless network will improve its throughput than without applying any standard. Authors also made comparative analysis of throughput obtained for topology consisting of different number of nodes with standard and without standard and ended up with the conclusion that throughput obtained with standard 802.11g is more than $802.11 \mathrm{~b}$.
\end{abstract}

Keywords: Audio Frequency, Radio Frequency, Wireless fidelity, unregulated signal frequency.

\section{INTRODUCTION}

Wireless technologies are becoming more and more popular around the world. Consumers appreciate the wireless lifestyle, relieving them of the well known "cable chaos" that tend to grow under their desk. Nowadays, the world would virtually stop if wireless communication suddenly became unavailable. Both our way of life and the global economy are highly dependent on the flow of information through wireless mediums like television and radio. Cell phones have become highly available during the last decade. [1]

New wireless technologies are introduced at an increasing rate. During the last few years the IEEE 802.11 technologies have started to spread rapidly, enabling consumers to set up their own wireless networks. This constitutes an important change in how wireless communications are made available to consumers.

Wireless networks are no longer provided by big corporations alone, they can just as well be implemented by individuals. Our society is becoming more and more dependent on wireless communications as new areas of use are introduced. [2]

People frequently think of the terms "wireless" and "Wi$\mathrm{Fi}^{\prime \prime}$ as synonymous, when they describe two entirely different ways of accessing the Internet. While a wireless connection utilizes a cellular network and allows you to use the Internet almost anywhere, establishing a Wi-Fi connection requires you to install hardware in your home or use your computer within range of a router to which you have access. Both connection types have benefits and drawbacks in areas such as transfer speed, signal range and cost [3]. Wi-Fi is a standard for short-distance wireless communication, used primarily by computers.

\section{A. $802.11 b$}

\section{WI-FI TECHNOLOGY}

802.11b has a maximum raw data rate of $11 \mathrm{Mbit} / \mathrm{s}$ and uses the same media access method defined in the original standard. The dramatic increase in throughput of $802.11 \mathrm{~b}$ (compared to the original standard) along with simultaneous substantial price reductions led to the rapid acceptance of $802.11 \mathrm{~b}$ as the definitive wireless LAN technology.

802.11b devices experience interference from other products operating in the $2.4 \mathrm{GHz}$ band. Devices operating in the $2.4 \mathrm{GHz}$ range include microwave ovens, Bluetooth devices, baby monitors, cordless telephones and some amateur radio equipment [3].

\section{B. $802.11 \mathrm{~g}$}

This works in the $2.4 \mathrm{GHz}$ band (like $802.11 \mathrm{~b}$ ), and uses OFDM based transmission scheme. It operates at a maximum physical layer bit rate of $54 \mathrm{Mbit} / \mathrm{s}$ exclusive of forward error correction codes, or about $22 \mathrm{Mbit} / \mathrm{s}$ average throughputs. $802.11 \mathrm{~g}$ hardware is fully backward compatible with $802.11 \mathrm{~b}$ hardware and therefore is encumbered with legacy issues that reduce throughput when compared to 802.11 a by $~ 21 \%$ [4].

Authors have done survey of energy efficient data gathering techniques for wireless sensor networks as a part of research work [5].

\section{COMPARATIVE STUDY}

\section{A. Simulation scenario and metrics}

In order to evaluate and validate the throughput of wireless and Wi-Fi connections along with its standards authors have implemented this experiment using NS-2 [6]. Authors have implemented topology for 4,5,6 and 7 nodes over an area of $500 \times 500 \mathrm{~m}$. All nodes have radio range of 
$\mathrm{R}=25 \mathrm{~m}$. Authors have considered wireless channel, Two Ray Ground Propogation model, Wireless Physical layer as network interface, type of queue is priority queue with queue length 50, media of communication is omni directional antenna and Routing protocol used is AODV. Table I shows details of simulation parameters.

TABLE I

SUMMARY OF SIMULATION PARAMETER

\begin{tabular}{|l|l|}
\hline \multicolumn{1}{|c|}{ Parameters } & \multicolumn{1}{c|}{ Value Description } \\
\hline Number of nodes (N) & $4,5,6,7$ \\
\hline Simulation area (A) & $500 X 500$ \\
\hline Wireless Radio Range (R) & $25 \mathrm{~m}$ \\
\hline Communication Channel & wireless \\
\hline Propagation & Two Ray Ground \\
\hline Network Interface & 802.11 MAC \\
\hline Type of queue & Priority queue \\
\hline Queue length & 50 \\
\hline Media to communicate & Omni Antenna \\
\hline Routing protocol & AODV \\
\hline
\end{tabular}

\section{B. Algorithm Approach}

Authors have written two different algorithms. First algorithm shown in Table II is for setting up topology with node mobility. Second algorithm shown in Table III is for calculating throughput for different topology with and without standard.

TABLE II

TOPOLOGY SETUP WITH NODE MOBILITY

Set default parameters

Create new instance of scheduler

Create trace file

Create name file

Configure all nodes

Create node and initialize position of the node

Design topology

Terminate simulation

\section{THROUGHTPUT CALCULATION}

Initialize parameter like received Size, Start Time, Stop

Time

Store start time

Update total received packet size and store packets arrival time

Rip of header

3.2 Store received packets size

Print Average Throughput in kbps.

$\mathrm{T}=$ (received Size/(Stop Time - Start

\section{Time) $) * 8 / 1000$}

\section{Simulation Methodology and Results}

Authors have implemented 4 different topologies with 4,5,6 and 7 nodes which are shown in Figure I to IV . Authors have considered Wi-Fi 802.11 without standard, Wi-Fi 802.11 with standard ' $b$ ' and ' $g$ '. The results were extracted for a specific time period over no. of generated packets. Throughput obtained in all three cases is listed in Table IV. Figure V shows throughput obtained for the topology without standard, with standards 802.11g and $802.11 \mathrm{~b}$

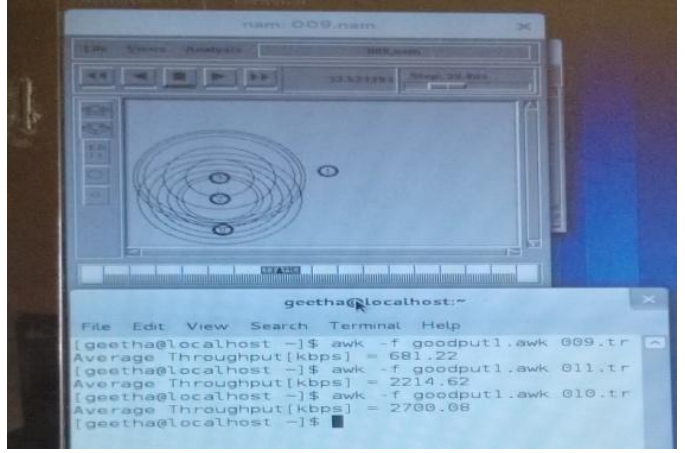

Figure 1. Simulation topology with 4 nodes

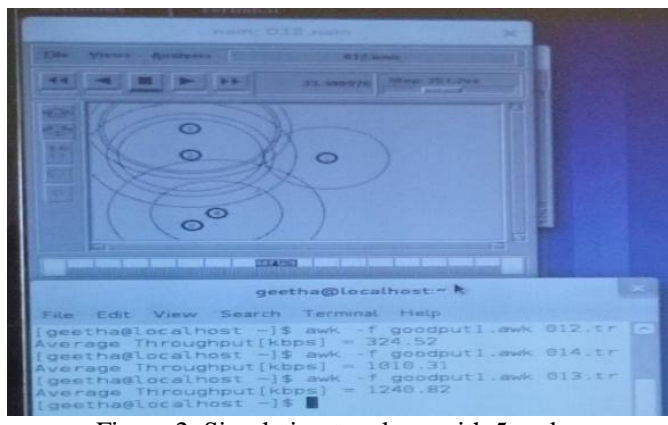

Figure 2. Simulation topology with 5 nodes

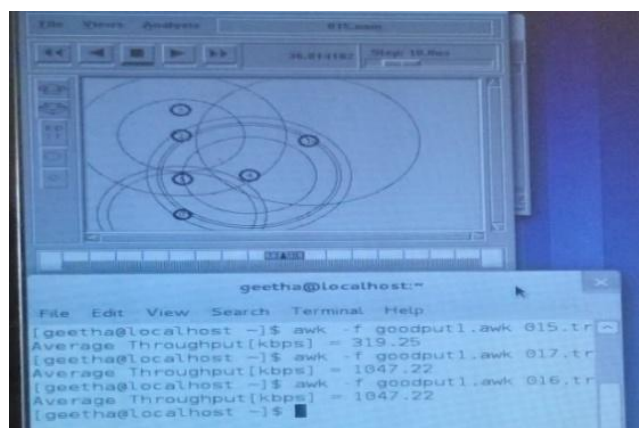

Figure 3. Simulation topology with 6 nodes

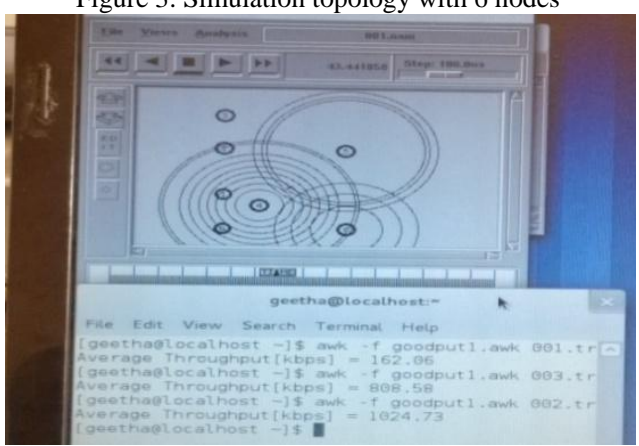

Figure 4. Simulation topology with 7 nodes

ABLE IIIII

THROUGHTPUT OBTAINED FOR THE TOPOLOGY WITHOUT STANDARD, WITH STANDARD 802.11g AND 802.11b

\begin{tabular}{|l|l|l|l|}
\hline $\begin{array}{c}\text { No. } \\
\text { of } \\
\text { nodes }\end{array}$ & $\begin{array}{c}\text { Throughput } \\
\text { without } \\
\text { standard } \\
\text { (kbps) }\end{array}$ & $\begin{array}{c}\text { Throughput } \\
\text { with standard } \\
\mathbf{8 0 2 . 1 1} \\
\text { (kbps) }\end{array}$ & $\begin{array}{c}\text { Throughput } \\
\text { with } \\
\text { Standard } \\
\mathbf{8 0 2 . 1 1 g} \\
\text { (kbps) }\end{array}$ \\
\hline 4 & 681.21 & 2700.08 & 2214.62 \\
\hline 5 & 324.52 & 1240.82 & 1010.31 \\
\hline 6 & 319.25 & 1047.22 & 1047.22 \\
\hline 7 & 162.06 & 1024.73 & 808.58 \\
\hline
\end{tabular}


Vol. 4, Issue 1, January 2015

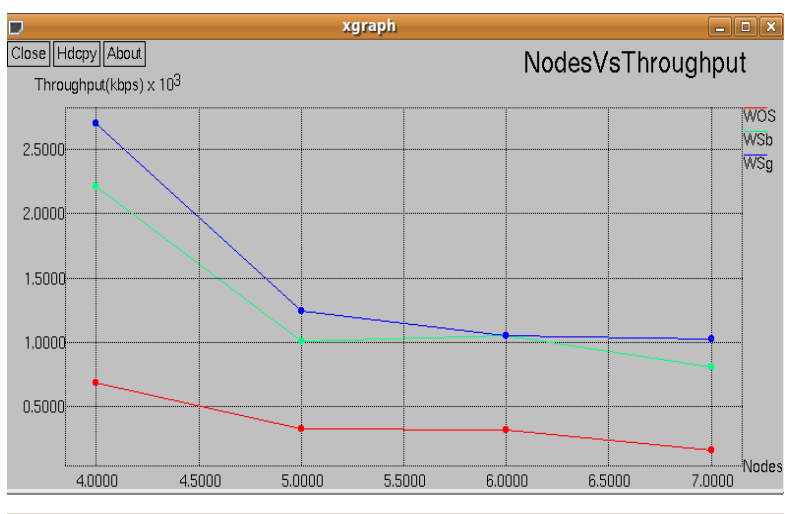

Figure 5. Simulation Result

\section{CONCLUSION AND FUTURE WORK}

According to the study and analysis of various wireless standards with a quantitative evaluation in terms of network topology and throughput, authors have ended up with two conclusions, Throughput obtained with any standard always more than throughput obtained without any standard in wireless environment. Comparison of $802.11 \mathrm{~g}$ with $802.11 \mathrm{~b}$ shows that throughput obtained with standard $802.11 \mathrm{~g}$ is more than $802.11 \mathrm{~b}$.Every wireless standard has its own application, advantage and drawbacks based on parameters. Here even though throughput of $802.11 \mathrm{~g}$ is more than $802.11 \mathrm{~b}$ the drawback is, appliances may interfere on the unregulated signal frequency due to which efficiency of the application may be compromised. In future authors have to work on this criteria.

\section{ACKNOWLEDGEMENT}

This work is supported in part by a grant from VGST, Govt. of Karnataka. The Author wish to thank VGST, Govt. of Karnataka for awarding Grant under K-Fist (Level-1) for the year 2012-14. The Authors are also thankful to The Management and The Principal of NIE, Mysore for their Encouragement in this regard.

\section{REFERENCES}

[1] Subhas C. Mukhopadhyay, Anuroup Gaddam and Gourab S. Gupta, "Wireless sensors for Home Monitoring", College of Science, Massey University, Palmerston North, Newzeland, Recent Patents on Electrical Engineering 2008

[2] M.S. Gast, "802.11 Wireless Networks", First Edition, O'Reilly, 2002.

[3] Frank Ohrtman, Konrad Roeder," Wi-Fi Handbook : Building 802.11b Wireless Networks Paperback “,April 10, 2003.

[4] Zeb Hallock, Suyog Deshpande, Roland Saville, "Cisco Connected Mobile Experiences (CMX)", September 4, 2014.

[5] Bhat Geetalaxmi Jayram , D. V. Ashoka," Merits and Demerits of Existing Energy Efficient Data Gathering Techniques for Wireless Sensor Networks", International Journal of Computer Applications (0975-8887), Vol. 66, Issue 9, March 2013.

[6] Trrrawat Issariyakul, Ekram Hossain, Introduction to Network Simulator NS2, Springer, New York, 2nd edition, 2012.

\section{BIOGRAPHIES}

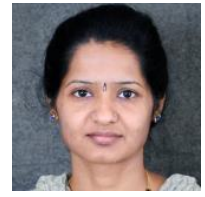

Bhat Geetalaxmi Jairam was born on 17th May 1975 and presently working as a Associate Professor, Department of Information Science and Engineering, National Institute of Engineering, Mysore.
She has obtained her Bachelor's degree in Electronics from Dr. Baba Saheb Ambedker University, Aurangabad then she has obtained her M.Tech from VTU and secured II-Rank in M-tech. Now she is pursuing $\mathrm{PhD}$ degree in Information Science and Engineering from VTU, University, Belgaum. Her registration year is NOVEMBER 2011. She is having more than 9 years of experience in Teaching. Her field of interest are Wireless Sensor Network, Computer Networks, Data Structures, Software Engineering, Graph Theory, Advanced Microprocessors, Electronics Devices and circuits. Her Research has got Grant along with citation award from, VGST (K-FIST-Level-I) citation award, Bangalore. She has presented four research papers in different international conferences, three papers in international journals and one in national conference awarded with best paper award.

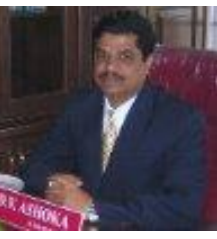

D. V. Ashok presently working as a Professor and Head, Department of Computer Science and Engineering, JSS Academy of Technical Education, Bangalore. He received his M.Tech from VTU and Ph.D degree in Computer Science and Engineering from Dr. MGR, University, Chennai. He has more than 19 years of academic, administrative and research experience. His fields of interest are Requirement Engineering, Knowledge Engineering, Operating System, Computer Organization, Software Architecture and Cloud Computing. He was published more than 35 research papers in national, international conferences and journals. He serves as member of technical program committee/editorial board of several national /International conferences and journals. He is also a member of FELLOW IEI, IEEE, MISTE, MCSI and MIAENG. His Biodata has been published in Marquis Who's Who in the World - 2011 Edition. Dr. DV Ashoka is one of the National Award winners "Rashtriya Ekta Samman-2013". 